\title{
Is there a role for vagus nerve stimulation in the treatment of posttraumatic stress disorder?
}

\author{
Christa K. McIntyre*,1 \\ ${ }^{1}$ Associate Professor of Cognition and Neuroscience, School of Behavioral and Brain Sciences, The University of Texas at Dallas, \\ Richardson, TX 75080, USA \\ *Author for correspondence: christa.mcintyre@utdallas.edu
}

First draft submitted: 6 March 2018; Accepted for publication: 23 March 2018; Published online: 25 May 2018

Keywords: adjunct $\bullet$ anxiety disorders $\bullet$ autonomic nervous system $\bullet$ cognitive behavior therapy $\bullet$ exposure therapy - extinction • memory • neural plasticity • neuromodulation • PTSD

Posttraumatic stress disorder (PTSD) develops in individuals who have been exposed to trauma and consequently suffer distress or functional impairment for at least 1 month. Symptoms include feelings of re-experiencing the traumatic event, avoiding reminders of the trauma, heightened anxiety and arousal, and negative thoughts or feelings. Recent natural disasters, mass shootings, terrorist attacks and cities under siege add to the global burden of PTSD which, according to a 2017 study, affects $4-6 \%$ of the global population [1], although the majority of traumas are related to accidents and sexual or physical violence [2]. Unfortunately, there is no known cure, and current treatments are not effective for all patients. A PTSD psychopharmacology working group recently published their consensus statement calling for immediate action to address the crisis in PTSD treatment, citing three major concerns. First, only two drugs (sertraline and paroxetine) are approved by the US FDA for the treatment of PTSD. These medications reduce symptom severity but may not produce complete remission of symptoms. The second concern is related to polypharmacy. PTSD patients are prescribed medications to address each of their many unique and diverse symptoms including anxiety, difficulty sleeping, sexual dysfunction, depression and chronic pain, with insufficient empirical investigations of drug interactions. The high comorbidity between PTSD and addiction provides further challenges for pharmacotherapies. The third major concern is the lack of advancements in the treatment of PTSD; no new medications have been approved since 2001 [3].

Going beyond symptom relief, the 'gold standard' trauma-focused approach to treating PTSD pathology is exposure-based therapy, where patients are exposed to the reminders of the trauma until they learn to associate these cues with safety. Although there is good evidence for effectiveness with this approach, not all patients fully respond to the therapy [4]. Exposure therapy depends on the process of extinguishing the conditioned fear memory, which is overcome by a new memory that develops through repeated exposures. The patients with anxiety disorders and PTSD show impairments in their ability to extinguish conditioned fears [5,6], which could contribute to the development of disorders and may interfere with progress in therapy. Because the memory of the trauma is not lost but, rather, improvements through therapy depend on new learned associations that compete with traumatic associations, the balance of the two memories can shift over time, leading to relapse [7]. Other challenges include the difficulty in recognizing and extinguishing fear of all conditioned stimuli, and a high dropout rate [8], which is not surprising given that avoidance is one of the symptoms of PTSD.

Many animal research labs have made efforts to develop adjunctive treatments to accelerate or enhance the effects of exposure-based therapies. Pioneering work carried out by Michael Davis showed that administration of the cognitive-enhancing drug $\mathrm{d}$-cycloserine before exposing rats to unreinforced conditioned cues enhanced extinction [9], and he and his colleagues subsequently translated the discovery when they found that $\mathrm{d}$-cycloserine also enhanced the effects of exposure therapy in patients with specific phobias [10]. However, results of studies assessing the effects of cognitive enhancers as adjuncts to exposure therapy are mixed in the case of PTSD [11,12]. A possible explanation is that drugs given before exposure therapy sessions run the risk of reinforcing negative associations if exposure produces anxiety. Anxiolytic drugs have been tried, based on evidence that these drugs should 
improve tolerability and reduce the anxiety response during exposure. However, results indicate that anxiolytic drugs do not enhance the effects of exposure therapy [12]. One explanation is that the anxiety response is required for success in exposure therapy because patients must learn not to fear their own fear response [13]. Alternatively, just as stress can enhance the storage of traumatic events, the anxiety response may enhance the consolidation of the extinction memory [14]. Consistent with this, anxiolytic drugs tend to impair memory consolidation. An ideal adjunct would tap into the mechanisms that enhance the consolidation of traumatic memories in order to promote extinction memories that are just as strong, all the while bypassing or avoiding the aversive stress response.

Emerging evidence suggests that vagus nerve stimulation (VNS) may be a beneficial adjunct to exposure-based therapies through its pairing-specific enhancement of memory consolidation and neural plasticity. Interest in the vagus nerve (the 10th cranial nerve) as a neuromodulator comes from several decades of research indicating that the vagus nerve serves as a bridge between the peripheral autonomic nervous system and the brain. It signals the brain during times of heightened sympathetic activity, promoting rapid storage of memories that are important for survival [15]. As part of the parasympathetic nervous system, activation of the vagus nerve counteracts the sympathetic stress response.

VNS enhances memory in rats and humans [16-18], suggesting that pairing VNS with unreinforced exposure to conditioned cues may enhance the consolidation of the extinction memory. Consistent with this hypothesis, we found that VNS enhanced extinction of conditioned fear in rats [19]. Extensive evidence indicates that VNS promotes neural plasticity, especially when it is paired with training [20,21], and this effect involves VNS modulation of the locus coeruleus noradrenergic system [22,23]. We have observed plasticity effects in the extinction-associated infralimbic prefrontal cortex - basolateral amygdala pathway after pairing VNS with exposure to unreinforced conditioned cues [24,25], suggesting that VNS-enhanced extinction may be robust, long lasting, and less susceptible to relapse. In a recent study, we found that VNS also enhanced extinction of conditioned fear in a rat model of PTSD. These rats express many of the biomarkers and behavioral phenotypes that are associated with PTSD and, importantly, they are resistant to extinction of conditioned fear [26,27]. We found that VNS administration during extinction sessions reversed this extinction impairment and prevented the return of fear. VNS-treated rats also performed better on tests of anxiety, arousal, avoidance, and social interactions 1 week later, indicating that reversal of the extinction impairment translated to improvements in other PTSD symptoms [28]. In addition, chronic, unpaired VNS, as is used in the treatment of epilepsy and depression, improved performance on the Hamilton Anxiety Scale in some patients with anxiety disorders [29], and reduced anxiety-like behavior in rats [30]. The effects of VNS on extinction in our studies are not seen when the VNS is administered $30 \mathrm{~min}$ to $1 \mathrm{~h}$ after training [19]. Therefore, VNS alone is not sufficient to reduce the fear response. These findings suggest that VNS may reduce anxiety, but pairing-specific plasticity and memory modulation is necessary for extinction enhancement. Our recent, unpublished findings indicate that rats are more likely to explore the open arms of an elevated plus maze immediately after receiving VNS, suggesting that VNS produces an acute anxiolytic effect. Furthermore, corticosterone levels increased significantly in sham-treated rats following testing on the elevated plus maze, but such an increase was not observed in VNS-treated rats. This work should be replicated in other contexts, but it is an encouraging first step toward identifying an adjunct therapy that may improve tolerability and efficacy in exposure-based therapies.

The US FDA approved VNS as a method to prevent seizures in treatment-resistant epilepsy patients in 1997, and in 2000 for treatment-resistant depression. The vagus nerve innervates the nucleus of the solitary tract, which projects to the locus coeruleus and other limbic and forebrain cortical areas [31,32]. VNS increases levels of monoamines in the brain [22,33-34] and the locus coeruleus plays a role in VNS-induced reduction of seizures [35]. The current clinical practice of VNS involves surgical implantation of an electrode that is attached to the left cervical vagus nerve through an incision in the neck. The electrode is connected by a lead that is tunneled under the skin to a pulse generator that is subcutaneously implanted in the chest. Surgical complications, such as infection or vocal cord effects, occur in about $1 \%$ of patients [36]. Less invasive approaches to stimulating the vagus nerve may be effective. In the 19th century, the neurologist James Leonard Corning developed devices to stimulate the vagus nerve transcutaneously, while compressing the carotid artery, and he observed a decrease in the frequency and duration of seizures [37]. These were not controlled studies and Corning abandoned the approach because of side effects such as dizziness and syncope; however, transcutaneous VNS (t-VNS) has recently regained status as a clinical tool. Noninvasive electrical stimulation of the vagus can be administered transcutaneously through the afferent auricular branch of the nerve with electrodes clipped to the concha region of the ear. With this t-VNS the electrical stimulus, with an intensity that is above sensory detection but below the pain threshold, is applied 
through the skin to the receptive field of the auricular branch. Mixed results were seen in a recent study examining $\mathrm{t}$-VNS effects on extinction of conditioned fear in humans [38] and clinical research on the use of t-VNS is limited, but it seems to be safe and well tolerated [39,40]. Transcutaneous versions of VNS may provide the benefits of VNS without the risks of surgery; however, t-VNS is not yet an established therapy and determination of its efficacy requires further investigation.

VNS holds promise as an adjunct to exposure-based therapies because it enhances memory consolidation and promotes synaptic plasticity while dampening the sympathetic stress response. Although VNS has been used in humans for over two decades, the practice of pairing it with exposure therapy has not been tested in patients and many questions remain unanswered. Eighty percent of the cervical fibers of the left vagus nerve are afferent sensory fibers [41] and preclinical studies are currently underway to examine the relative contributions of PNS versus CNS effects of VNS. Individual differences in the nerve and in arousal state may cause variability in effects in human patients. Identification of a reliable biomarker for VNS effects would be beneficial for customizing parameters in treatment across individuals, and it may be used to gauge the potential effectiveness of less invasive methods of stimulating the vagus nerve such as t-VNS. Finally, it remains to be determined whether VNS has an acute anxiolytic effect. According to our model, stimulation of the vagus nerve bypasses the sympathetic response to threat, while still promoting plasticity and rapid consolidation of long-lasting memories. The role of the vagus nerve in the parasympathetic nervous system is to slow the sympathetic stress response. Some evidence indicates that chronic VNS reduces anxiety in humans and in rats. If VNS can immediately reduce anxiety, this may, or may not be beneficial for exposure-based therapies. It may interfere with the opportunity to extinguish the fear of the fear response. Conversely, it may hasten progress and improve compliance by severing the association between exposure to trauma cues and the conditioned fear response during therapy. Studies are currently underway to determine whether different stimulation parameters can be used to dissociate memory effects of VNS from anxiolytic effects.

During critical periods in development, the brain is more plastic than it is later in life. However, when under pressure, the adult brain can adapt. Rapidly stored, long-lasting memories of emotionally arousing events are an example of robust neural plasticity that can be accomplished in the adult brain. In 1890, William James wrote, "An experience may be so exciting emotionally as almost to leave a scar on the cerebral tissues". The neural plasticity that underlies traumatic memories can be adaptive, reducing the likelihood that a dangerous behavior will be repeated. Occasionally, traumatic memories have maladaptive consequences, leading to anxiety- or stress-related disorders. We aim to harness the potential of the vagus nerve to drive neural plasticity during exposure therapy, while concurrently interrupting the sympathetic fight-or-flight response. If successful, we will take advantage of mechanisms that exist for the purpose of leaving a lasting impression on the brain in order to heal the cerebral scars left by trauma.

\section{Acknowledgements}

The author thanks Dr Rimenez Souza and Dr Lindsey Noble for thoughtful comments on a draft of this article.

\section{Financial \& competing interests disclosure}

The author's research is supported by the Defense Advanced Research Projects Agency (DARPA) Biological Technologies Office (BTPO) Electrical Prescriptions (ElectRx) program under the auspices of Dr Doug Weber through the Space and Naval Warfare System Center, Pacific. Grant/Contract number DARPA-AA-14-38 and DARPA-BAA-15-06 and the NIMH, MH 105014. The author is a holder of a patent entitled 'Enhancing Fear Extinction using Vagus Nerve Stimulation'. The authors have no other relevant affiliations or financial involvement with any organization or entity with a financial interest in or financial conflict with the subject matter or materials discussed in the manuscript apart from those disclosed.

No writing assistance was utilized in the production of this manuscript.

\section{References}

1. Koenen KC, Sumner JA, Gilsanz P et al. Post-traumatic stress disorder and cardiometabolic disease: improving causal inference to inform practice. Psychol. Med. 47(2), 209-225 (2017).

2. Kessler RC, Aguilar-Gaxiola S, Alonso J et al. Trauma and PTSD in the WHO World Mental Health Surveys. Eur. J. Psychotraumatol. 8(sup5), 1353383 (2017).

3. Krystal JH, Davis LL, Neylan TC et al. It is time to address the crisis in the pharmacotherapy of post-traumatic stress disorder: a consensus statement of the PTSD Psychopharmacology Working Group. Biol. Psychiatry 82(7), e51-e59 (2017). 
4. Powers MB, Halpern JM, Ferenschak MP, Gillihan SJ, Foa EB. A meta-analytic review of prolonged exposure for post-traumatic stress disorder. Clin. Psychol. Rev. 30(6), 635-641 (2010).

5. Anderson KC, Insel TR. The promise of extinction research for the prevention and treatment of anxiety disorders. Biol. Psychiatry 60(4), 319-321 (2006).

6. Milad MR, Orr SP, Lasko NB, Chang Y, Rauch SL, Pitman RK. Presence and acquired origin of reduced recall for fear extinction in PTSD: results of a twin study. J. Psychiatr. Res. 42(7), 515-520 (2008).

7. Boschen MJ, Neumann DL, Waters AM. Relapse of successfully treated anxiety and fear: theoretical issues and recommendations for clinical practice. Aust. N. Z. J. Psychiatry 43(2), 89-100 (2009).

8. Schottenbauer MA, Glass CR, Arnkoff DB, Tendick V, Gray SH. Nonresponse and dropout rates in outcome studies on PTSD: review and methodological considerations. Psychiatry 2008 71(2), 134-168 (2009).

9. Walker DL, Ressler KJ, Lu KT, Davis M. Facilitation of conditioned fear extinction by systemic administration or intra-amygdala infusions of D-cycloserine as assessed with fear-potentiated startle in rats. J. Neurosci. 22(6), 2343-2351 (2002).

10. Davis M, Ressler K, Rothbaum BO, Richardson R. Effects of D-cycloserine on extinction: translation from preclinical to clinical work. Biol. Psychiatry 60(4), 369-375 (2006).

11. Litz BT, Salters-Pedneault K, Steenkamp MM et al. A randomized placebo-controlled trial of D-cycloserine and exposure therapy for post-traumatic stress disorder. J. Psychiatr. Res. 46(9), 1184-1190 (2012).

12. Rothbaum BO, Price M, Jovanovic T et al. A randomized, double-blind evaluation of D-cycloserine or alprazolam combined with virtual reality exposure therapy for posttraumatic stress disorder in Iraq and Afghanistan war veterans. Am. J. Psychiatry 171(6), 640-648 (2014).

13. Tuerk PW, Wangelin BC, Powers MB et al. Augmenting treatment efficiency in exposure therapy for PTSD: a randomized double-blind placebo-controlled trial of yohimbine HCl. Cogn. Behav. Ther. 15, 1-21 (2018). (Epub ahead of print).

14. LaLumiere RT, McGaugh JL, McIntyre CK. Emotional modulation of learning and memory: pharmacological implications. Pharmacol. Rev. 69(3), 236-255 (2017).

15. McIntyre CK, McGaugh JL, Williams CL. Interacting brain systems modulate memory consolidation. Neurosci. Biobehav. Rev. 36(7), 1750-1762 (2012).

16. Clark KB, Krahl SE, Smith DC, Jensen RA. Post-training unilateral vagal stimulation enhances retention performance in the rat. Neurobiol. Learn Mem. 63(3), 213-216 (1995).

17. Clark KB, Smith DC, Hassert DL, Browning RA, Naritoku DK, Jensen RA. Posttraining electrical stimulation of vagal afferents with concomitant vagal efferent inactivation enhances memory storage processes in the rat. Neurobiol. Learn Mem. 70(3), 364-373 (1998)

18. Clark KB, Naritoku DK, Smith DC, Browning RA, Jensen RA. Enhanced recognition memory following vagus nerve stimulation in human subjects. Nat. Neurosci. 2(1), 94-98 (1999).

19. Peña DF, Engineer ND, McIntyre CK. Rapid remission of conditioned fear expression with extinction training paired with vagus nerve stimulation. Biol. Psychiatry 73(11), 1071-1077 (2013).

20. Hays SA. Enhancing rehabilitative therapies with vagus nerve stimulation. Neurotherapeutics 13(2), 382-394 (2016).

21. Hays SA, Rennaker RL, Kilgard MP. Targeting plasticity with vagus nerve stimulation to treat neurological disease. Prog. Brain Res. 207, 275-299 (2013).

22. Hassert DL, Miyashita T, Williams CL. The effects of peripheral vagal nerve stimulation at a memory-modulating intensity on norepinephrine output in the basolateral amygdala. Behav. Neurosci. 118(1), 79-88 (2004).

23. Hulsey DR, Riley JR, Loerwald KW, Rennaker RL, Kilgard MP, Hays SA. Parametric characterization of neural activity in the locus coeruleus in response to vagus nerve stimulation. Exp. Neurol. 289, 21-30 (2017).

24. Реп̆а DF, Childs JE, Willett S, Vital A, McIntyre CK, Kroener S. Vagus nerve stimulation enhances extinction of conditioned fear and modulates plasticity in the pathway from the ventromedial prefrontal cortex to the amygdala. Front. Behav. Neurosci. 8, 327 (2014).

25. Alvarez-Dieppa AC, Griffin K, Cavalier S, McIntyre CK. Vagus nerve stimulation enhances extinction of conditioned fear in rats and modulates Arc Protein, CaMKII, and GluN2B-Containing NMDA receptors in the basolateral amygdala. Neural Plast. 2016, 4273280 (2016).

26. Knox D, George SA, Fitzpatrick CJ, Rabinak CA, Maren S, Liberzon I. Single prolonged stress disrupts retention of extinguished fear in rats. Learn Mem. 19(2), 43-49 (2012).

27. Souza RR, Noble LJ, McIntyre CK. Using the single prolonged stress model to examine the pathophysiology of PTSD. Front. Pharmacol. 8, 615 (2017).

28. Noble LJ, Gonzalez IJ, Meruva VB et al. Effects of vagus nerve stimulation on extinction of conditioned fear and post-traumatic stress disorder symptoms in rats. Transl. Psychiatry 7(8), e1217 (2017).

29. George MS, Ward HE, Ninan PT et al. A pilot study of vagus nerve stimulation (VNS) for treatment-resistant anxiety disorders. Brain Stimul. 1(2), 112-121 (2008).

30. Furmaga H, Shah A, Frazer A. Serotonergic and noradrenergic pathways are required for the anxiolytic-like and antidepressant-like behavioral effects of repeated vagal nerve stimulation in rats. Biol. Psychiatry 70(10), 937-945 (2011). 
31. Nemeroff CB, Mayberg HS, Krahl SE et al. VNS therapy in treatment-resistant depression: clinical evidence and putative neurobiological mechanisms. Neuropsychopharmacology 31(7), 1345-1355 (2006).

32. Henry TR, Bakay RA, Pennell PB, Epstein CM, Votaw JR. Brain blood-flow alterations induced by therapeutic vagus nerve stimulation in partial epilepsy: II. prolonged effects at high and low levels of stimulation. Epilepsia 45(9), 1064-1070 (2004).

33. Manta S, Dong J, Debonnel G, Blier P. Enhancement of the function of rat serotonin and norepinephrine neurons by sustained vagus nerve stimulation. J. Psychiatry Neurosci. 34(4), 272-280 (2009).

34. Roosevelt RW, Smith DC, Clough RW, Jensen RA, Browning RA. Increased extracellular concentrations of norepinephrine in cortex and hippocampus following vagus nerve stimulation in the rat. Brain Res. 1119(1), 124-132 (2006).

35. Krahl SE, Clark KB, Smith DC, Browning RA. Locus coeruleus lesions suppress the seizure-attenuating effects of vagus nerve stimulation. Epilepsia 39(7), 709-714 (1998).

36. Howland RH. Vagus nerve stimulation. Curr. Behav. Neurosci. Rep. 1(2), 64-73 (2014).

37. Lanska DJ. J.L. Corning and vagal nerve stimulation for seizures in the 1880s. Neurology 58(3), 452-459 (2002).

38. Burger AM, Verkuil B, Fenlon $\mathrm{H}$ et al. Mixed evidence for the potential of non-invasive transcutaneous vagal nerve stimulation to improve the extinction and retention of fear. Behav. Ther. 97, 64-74 (2017).

39. Stefan H, Kreiselmeyer G, Kerling F et al. Transcutaneous vagus nerve stimulation (t-VNS) in pharmacoresistant epilepsies: a proof of concept trial. Epilepsia 53(7), e115-e118 (2012).

40. Lamb DG, Porges EC, Lewis GF, Williamson JB. Non-invasive vagal nerve stimulation effects on hyperarousal and autonomic state in patients with post-traumatic stress disorder and history of mild traumatic brain injury: preliminary evidence. Front. Med. (Lausanne) 4 , 124 (2017).

41. Agostoni E, Chinnock JE, De Daly MB, Murray JG. Functional and histological studies of the vagus nerve and its branches to the heart, lungs and abdominal viscera in the cat. J. Physiol. 135(1), 182-205 (1957). 
